\title{
Rediscovery of Plesiocolochirus inornatus (Marenzeller, 1881) (Echinodermata: Holothuroidea: Dendrochirotida: Cucumariidae) in Japan and new information on its external and ossicle morphology
}

\author{
Yusuke YAMANA $^{1, *} \&$ HisAnORI KOHTSUKA ${ }^{2}$ \\ ${ }^{1}$ Wakayama Prefectural Museum of Natural History, 370-1 Funo, Kainan, Wakayama 642-0001, Japan \\ ${ }^{2}$ Misaki Marine Biological Station, School of Science, University of Tokyo, 1024 Koajiro, Misaki, Miura, Kanagawa 238-0225, \\ Japan
}

Received 16 September 2015; Accepted 22 March 2016 Responsible Editor: Shigeaki Kojima

\begin{abstract}
The cucumariid sea cucumber Plesiocolochirus inornatus (Marenzeller, 1881) was rediscovered in shallow water of the Pacific Ocean, on the south coast of Fukushima Prefecture, eastern Japan. The last report of this species in Japanese waters was published a hundred years ago, although this species possibly has a wide distribution in Japanese waters. Since there is no detailed information on its external appearance, we prepared images of the body shape on the basis of six new specimens. The body of $P$. inornatus has obviously distinguishable dorsal and ventral sides in the living state with tapered ends that turn upwards, mid-body of the ventral side extremely flattened and nearly half-round in cross-section, dorsal surface smooth with retracted minute ambulacral-papillae scattered uniformly regardless of radii or inter-radii. Furthermore, detailed images of ossicle shape and calcareous ring shape were prepared.
\end{abstract}

Key words: Colochirus, Cucumariidae, Dendrochirotida, Holothuroidea, Plesiocolochirus inornatus

\section{Introduction}

After the report of one small specimen in northern Kyushu by the U.S. Fish Commission Steamer Albatross (Ohshima 1914, 1918), the cucumariid sea cucumber Plesiocolochirus inornatus (Marenzeller 1881, as Colochirus inornatus) has been forgotten with respect to Japanese benthology.

Marenzeller (1881) published a very detailed description for this species. However, he provided only a few sketches of the ossicles and no sketches of the body shape and calcareous ring shape. Unfortunately, images of the body shape for $P$. inornatus are also rarely available in the literature (e.g. Mitsukuri 1912, Yang 1937, as Pentacta inornatus, Liao and Clark 1995, as Acolochirus inornatus), and all the images are unclear and indiscernible. Thus, obvious images to support the original description of Marenzeller (1881) have not been available to date.

Recently, we collected a cucumariid sea cucumber from

*Corresponding author: Yusuke Yamana; E-mail, yamanamako@gmail. com a shallow water of the Pacific Ocean, on the south coast of Fukushima Prefecture, eastern Japan. It is a very common and dominant species of the site. The specimen's living body shape showed its unique characteristics, and was not cylindrical like most cucumariid species. However, its living body shape was consistent with that of P. inornatus (Marenzeller 1881). For promoting biological research on this possibly common animal and taxonomical research for its congeners, here we have reported the morphology of this species by preparing detailed images of its body shape, ossicle shape, and shape of the calcareous ring.

\section{Materials and Methods}

On 15 November 2014, six individuals were collected using SCUBA from 5-6 m depth in the Pacific Ocean, by H. Kohtsuka, one of the authors, on the quay wall in front of the Environmental Aquarium Aquamarine Fukushima $\left(36^{\circ} 56^{\prime} 33^{\prime \prime} \mathrm{N}, 140^{\circ} 54^{\prime} 08^{\prime} \mathrm{E}\right)$ in Port Onahama, south coast of Fukushima Prefecture, Japan.

We fixed three of the specimens in $80 \%$ ethanol after anaesthetization; the remaining three were fixed without 

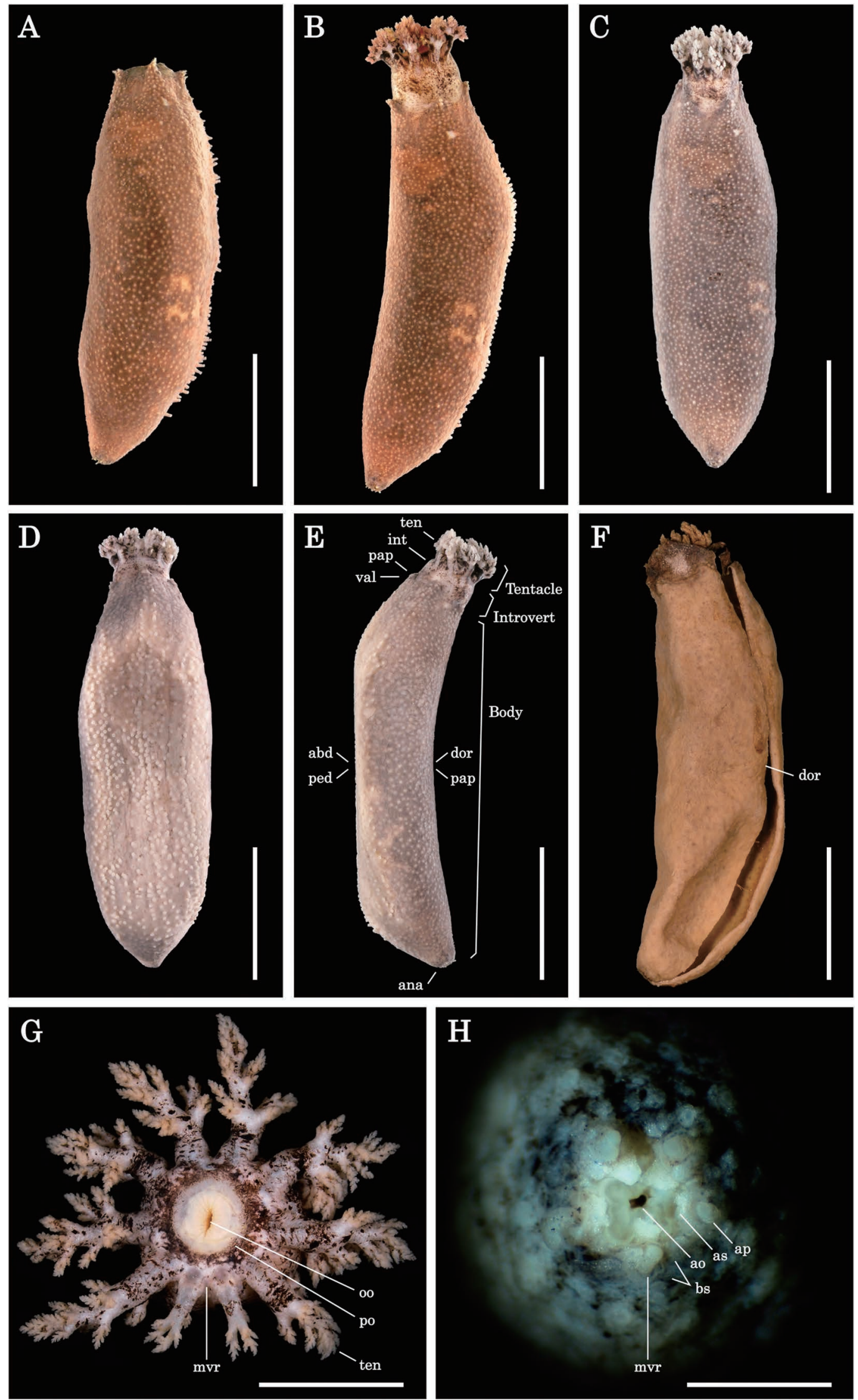
anaesthesia. Anaesthetization was performed in a menthol solution: $0.37 \mathrm{~g}$ of menthol was dissolved into $100 \mathrm{ml}$ of $99 \%$ ethanol, which was diluted to $3 \%$ with seawater. The specimens were immersed in the diluted solution for several hours; then, the tentacles emerged in response to a gentle squeeze of the body. Before fixation, magnesium chloride $\left(\mathrm{MgCl}_{2} \cdot 6 \mathrm{H}_{2} \mathrm{O}\right)$ was added to the solution at a ratio of $3 \mathrm{~g}$ per $100 \mathrm{ml}$ for further anaesthetization.

All six specimens were dissected, and the tentacles, anal appendages, Polian vesicles, stone canals, and calcareous rings were examined under a stereoscopic microscope (Nikon SMZ). To observe the morphology of the ossicles, small pieces of tissue were isolated (from each specimen) from the following parts of the body (Fig. 1E, G, H): 1) body-wall integument in the middle part of the abdominal side; 2) pedicels in the middle part of the abdominal side; 3) body-wall integument in the middle part of the dorsal side; 4) ambulacral-papillae in the middle part of the dorsal side; 5) body-wall integument and 6) papillae at the tip of the valve (body-wall projection in the anterior body end) of the abdominal-lateral side; 7) body-wall integument and 8) papilla in the anal periphery of the abdominal-lateral side; 9) tentacle on the abdominal-lateral side; 10) peri-oral skin on the abdominal-lateral side; and 11) middle part of the introvert on the abdominal-lateral side. Because no obvious differences were found among the ossicles of part 1 of the six specimens examined, examination of the ossicles of parts 2-11 were performed for only three specimens.

We dissolved the tissue samples in sodium hypochlorite solution $(\mathrm{NaClO}, 5 \%)$ under a compound microscope (Nikon Optiphot). Thus, even if ossicles from other parts were contaminated, we could judge from where those ossicles were isolated. The ossicles of three specimens were counted and measured on the basis of photographs. For every tissue sample, we measured a total of 13-200 ossicles to the nearest micrometer, depending on their density. The collected specimens and glass slides of their ossicles have been deposited in the Invertebrate Collection (INV) of the Wakayama Prefectural Museum of Natural History (WMNH), Kainan, Wakayama, Japan.

\section{Comparative material}

We also examined one of the syntypes of Plesiocolochirus inornatus (Marenzeller, 1881, as Colochirus inorna$t u s$ ). It is lodged in the Naturhistorisches Museum Wien (MNW), Wien, Austria (Jangoux and De Ridder, 1990). It was dissected by Marenzeller, and further dissection was not necessary (Fig. 1F). For observation of the ossicles of this specimen a small tissue sample was taken from the edge of Marenzeller's dissection cut in the mid-body wall of the specimen.

\section{Results \\ Plesiocolochirus inornatus (Marenzeller, 1881)}

(Figs. 1-6)

Colochirus inornatus Marenzeller, 1881: 130-132, Tafel. V. fig. 7, 7A; Lampert 1885: 127; Théel 1886: 77-78, 120, Pl. VI. fig. 8.; Mitsukuri 1912: 221-224, Pl. VIII. fig. 73. textfig. 40; Pearson 1910: 187; Ohshima 1914: 267; Ohshima 1918: 355-357, fig. 68; Imaoka 1995: 558.

Acolochirus inornatus (Marenzeller, 1881): Liao and Clark 1995: 473, fig. 284.

Pentacta inornata (Marenzeller, 1881): Chang 1937 (1948): 75-76.

Pentacta inornatus (Marenzeller, 1881): Yang 1937: 12 14, Pl. III. fig. 5, textfig. 7.

Plesiocolochirus inornatus (Marenzeller, 1881): Clark and Rowe 1971: 192-193.

\section{Material examined}

WMNH-2015-INV-1 (anaesthetized; length $57 \mathrm{~mm}$, width $20 \mathrm{~mm}$ ); WMNH-2015-INV-2 (anaesthetized; length $54 \mathrm{~mm}$, width $18 \mathrm{~mm}$ ); WMNH-2015-INV-3 (anaesthetized; length $61 \mathrm{~mm}$, width $14 \mathrm{~mm}$ ); WMNH-2015-INV-4 (unanaesthetized; length $45 \mathrm{~mm}$, width $15 \mathrm{~mm}$ ); WMNH2015-INV-5 (unanaesthetized; length $46 \mathrm{~mm}$, width $14 \mathrm{~mm}$ ); WMNH-2015-INV-6 (unanaesthetized; length $38 \mathrm{~mm}$, width $14 \mathrm{~mm}$ );

\section{Description}

Body with obviously distinguishable dorsal and ventral sides (Fig. 1A-E), mid-body of ventral side extremely flattened, and dorsal side cylindrical in shape, with tapered ends (both ends) that turned upwards. Mouth and anus located in anterior and posterior regions, respectively. Five developed radial oral 'valves' (body wall projection in anterior body end, which has role of shield for introvert and tentacles due to five valves in contact with each other), each valve with 2-6 non-retractile ambulacral-papillae. Outer layer of body integument inflexible and brittle. Body colour, light brown or greyish in dorsal, faint colour in mid-body of ventral side. Anterior tips of valves sometimes pigmented with deep brown or black. Mid-body surface not containing large calcareous plates called 'scales.'

Ten dendritic tentacles arranged in a single circle; two

Fig. 1. Plesiocolochirus inornatus (Marenzeller, 1881), dorsal views, A-C, abdominal view, D, lateral view, E, WMNH-2015-INV-1. A, $\mathrm{B}$, living state, before (A) and after (B) anaesthetization; C-E, preserved state, fixed with anaesthesia; dorsal view, F, MNW-11494, syntype. Scale bars: A-F, $20 \mathrm{~mm}$. Abbreviations indicating sites of tissue sampling for ossicle examination: abd, middle part of body on abdominal side; ana, anal periphery; dor, middle part of body on dorsal side; int, middle part of introvert; pap, ambulacral-papillae; ped, pedicels; ten, tentacle; val, valve (body wall projection in anterior body end). G and H, WMNH-2015-INV-3, front view of oral opening and circumoral tentacles $(\mathrm{G})$, front view of anal opening and anal appendages $(\mathrm{H})$. Scale bars: $\mathrm{G}, 10 \mathrm{~mm}$; H, $2 \mathrm{~mm}$. Abbreviations: ao, anal opening; ap, anal ambulacural-papilla; as, anal scale; bs, body scale; ten, tentacle; mvr, mid-ventral radial part; oo, oral opening; po, peri-oral part. 
Table 1. Summary of measurements and counts for six new specimens and one syntype of Plesiocolochirus inornatus (Marenzeller, 1881).

\begin{tabular}{|c|c|c|c|c|c|c|c|c|c|c|c|c|}
\hline \multirow{3}{*}{ Registration No. ${ }^{a}$} & \multirow{3}{*}{$\begin{array}{l}\text { Body } \\
\text { state }\end{array}$} & \multirow{3}{*}{$\begin{array}{l}\text { Gonad } \\
\text { state }\end{array}$} & \multirow{2}{*}{\multicolumn{2}{|c|}{$\begin{array}{l}\text { Body size } \\
(\mathrm{mm})\end{array}$}} & \multicolumn{8}{|c|}{ Count } \\
\hline & & & & & \multicolumn{2}{|c|}{$\begin{array}{l}\text { Pedicels } \\
\text { in a row }{ }^{b}\end{array}$} & \multirow{2}{*}{ Tentacles } & \multirow{2}{*}{$\begin{array}{c}\text { Valve } \\
\text { papillae }\end{array}$} & \multirow{2}{*}{$\begin{array}{c}\text { Anal } \\
\text { papillae }\end{array}$} & \multirow{2}{*}{$\begin{array}{l}\text { Anal } \\
\text { scales }\end{array}$} & \multirow{2}{*}{$\begin{array}{l}\text { Stone } \\
\text { canal }\end{array}$} & \multirow{2}{*}{$\begin{array}{l}\text { Polian } \\
\text { vesicle }\end{array}$} \\
\hline & & & Length & Width & Body & Introvert & & & & & & \\
\hline WMNH-2015-INV-1 & relax & testis & 57 & 20 & 85 & 0 & 10 & $2-4$ & 5 & 5 & 1 & 1 \\
\hline WMNH-2015-INV-2 & relax & ovarium & 54 & 18 & 82 & 0 & 10 & $3-5$ & 5 & 5 & 1 & 1 \\
\hline WMNH-2015-INV-3 & relax & testis & 61 & 14 & 89 & 0 & 10 & $3-5$ & 5 & 5 & 1 & 1 \\
\hline WMNH-2015-INV-4 & contract & ovarium & 45 & 15 & $?$ & 0 & 10 & $3-6$ & 5 & 5 & 1 & 1 \\
\hline WMNH-2015-INV-5 & contract & testis & 46 & 14 & $?$ & 0 & 10 & $3-5$ & 7 & 5 & 1 & 1 \\
\hline WMNH-2015-INV-6 & contract & ovarium & 38 & 14 & $?$ & 0 & 10 & $3-5$ & 5 & 5 & 1 & 1 \\
\hline MNW-11494 (syntype) & contract & ovarium & 65 & 19 & $?$ & 0 & 10 & $3-6$ & 5 & 5 & 1 & 1 \\
\hline
\end{tabular}

${ }^{a}$ WMNH: Wakayama Prefectural Museum of Natural History, MNV: Naturhistorisches Museum Wien. ${ }^{\mathrm{b}}$ Number of pedicels in a row along the longitudinal muscle in the mid-ventral radial zone of the abdominal side.

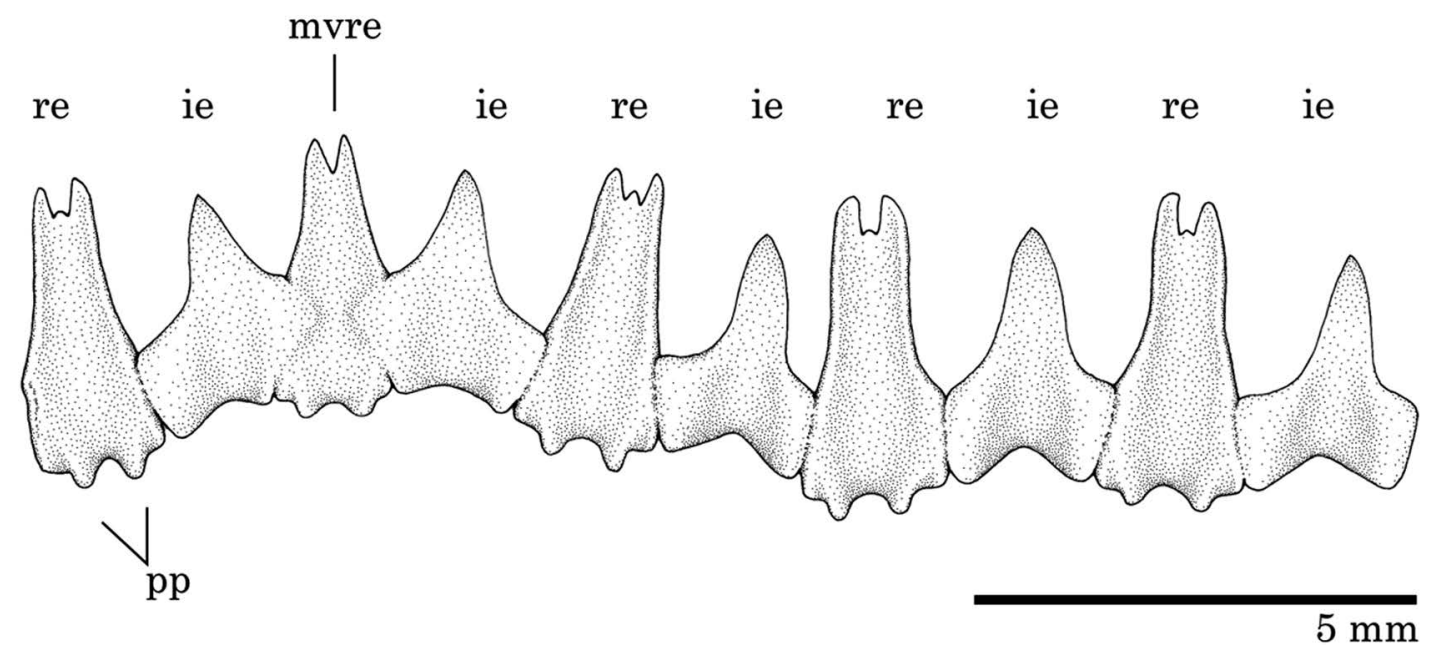

Fig. 2. Plesiocolochirus inornatus (Marenzeller, 1881), elements of calcareous rings, viewed from body cavity. Abbreviations: ie, interradial element; mvre, mid-ventral radial element; pp, posterior prolongation; re, radial element. WMNH-2015-INV-4.

tentacles on ventral side smaller (Fig. 1G). Colour of tentacles and introvert light brown or whitish brown or whitish grey, and generally variegated with blackish-brown.

Numerous retractile ambulacral-papillae scattering on dorsal side at constant density, regardless of radii or interradii. On abdominal side, pedicels form obscure three obscure bands along radii, not dense in inter-radii; on each side of thin longitudinal muscles, pedicels or ambulacralpapillae form an obscure, contiguous row (Fig. 1D, Table 1). Colour of pedicels varied depending on specimen, generally almost same or more faint than that of integument of adjacent body. Introvert region displayed no pedicels or ambulacral-papillae.

Around anus, five small scales (anal teeth) and five nonretractile ambulacural-papillae on most radii (Fig. 1H, Table 1). Anal scale (anal tooth) containing long calcareous structure. Many small scales covering body surface very restricted in anal periphery.

Calcareous ring solid, both radial and inter-radial ele- ments with fine tapering anterior projections, radial elements displayed terminal splits at anterior ends and prolongations at posterior ends. Prolongations slight, sometimes could not be determined. Inter-radial elements with wide indentations at posterior ends, and one radial element and two inter-radial elements of mid-abdominal side united together (Fig. 2). All specimens with one Polian vesicle and one stone canal (Table 1). Tubules of gonads mostly unbranched.

Four types of ossicles found in body-wall integument in middle part of abdominal and dorsal sides: mostly 1) 'cups' built with an $\mathrm{x}$-shaped central frame, 2) knobbed plates (some of which very thickening and forming 'knobbed button', mostly from inner layer of integument), widely varied in size, 3) poorly knobbed plates, 4) and multi-layered knobbed plates (Fig. 3, Table 2). Former two types, however, continuous with gradual intermediate forms in between; these ossicles, irrespective of forms, length 32-249 $\mu \mathrm{m}$ (mean $98-108 \mu \mathrm{m}$ ) in abdominal side and 


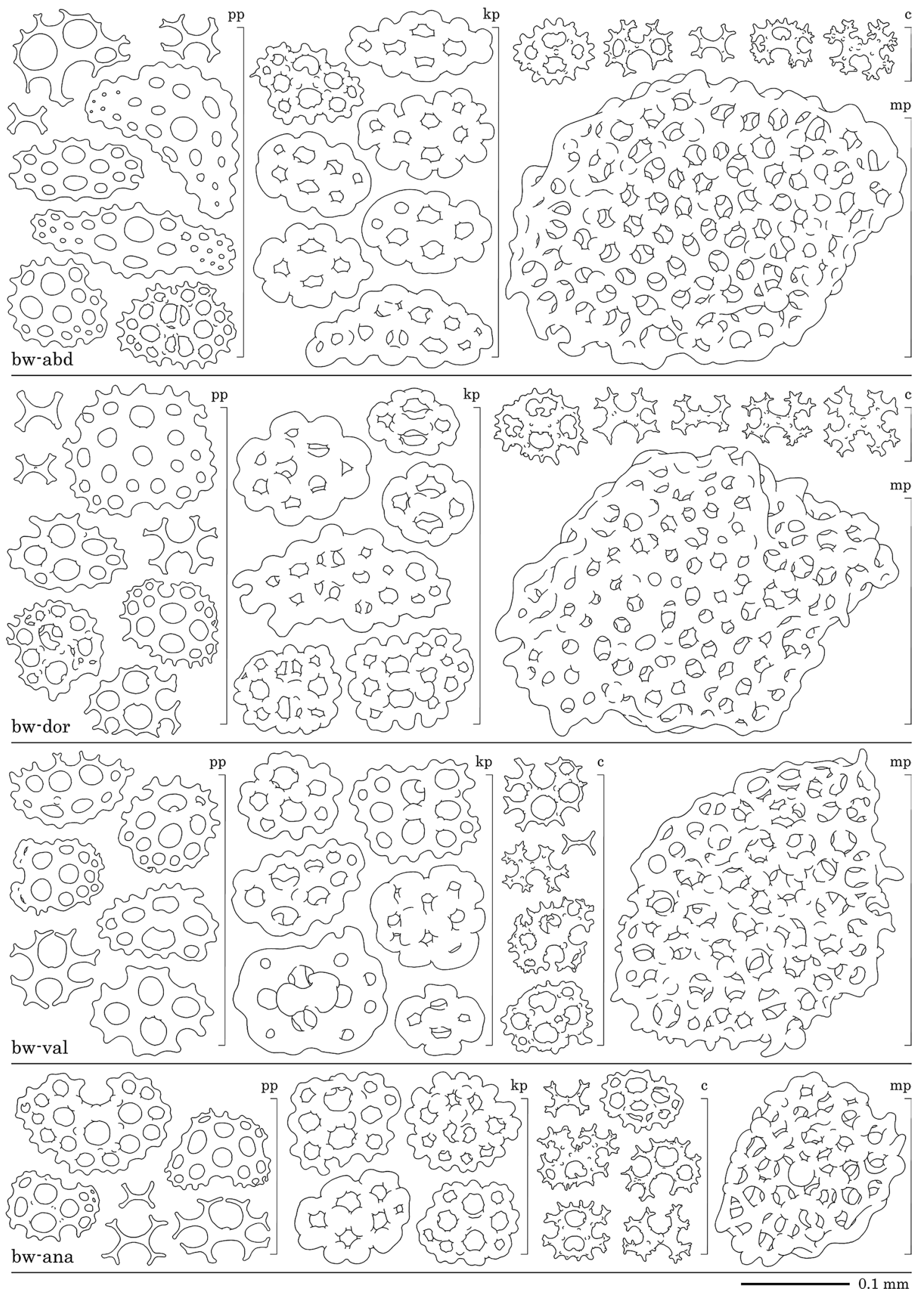

Fig. 3. Plesiocolochirus inornatus (Marenzeller, 1881), ossicles of body-wall integument in middle part of abdominal side (bw-abd), dorsal side (bw-dor), valve (bw-val) of abdominal-lateral side, and anal (bw-ana) of abdominal-lateral side. Abbreviations: c, cup; kp, knobbed plate; mp, multi-layered knobbed plate; pp, poorly knobbed plate. WMNH-2015-INV-4. 
Table 2. Measurements $(\mu \mathrm{m})$ of ossicles from body-wall integument, pedicels, and ambulacral-papillae of mid-body of Plesiocolochirus inornatus (Marenzeller, 1881) (Figs. 3, 4, and 6).

\begin{tabular}{|c|c|c|c|c|c|c|c|c|c|c|}
\hline \multirow{4}{*}{ Ossicle type } & \multicolumn{5}{|c|}{ Abdominal side (middle part) } & \multicolumn{5}{|c|}{ Dorsal side (middle part) } \\
\hline & \multicolumn{2}{|c|}{$\begin{array}{l}\text { Body-wall } \\
\text { integument }\end{array}$} & \multicolumn{3}{|c|}{ Pedicels } & \multicolumn{2}{|c|}{$\begin{array}{l}\text { Body-wall } \\
\text { integument }\end{array}$} & \multicolumn{3}{|c|}{ Ambulacral-papillae } \\
\hline & \multirow[b]{2}{*}{$(n)^{\mathrm{a}}$} & Length & \multirow[b]{2}{*}{$(n)^{\mathrm{a}}$} & Length & Holes $^{\mathrm{b}}$ & \multirow[b]{2}{*}{$(n)^{\mathrm{a}}$} & Length & \multirow[b]{2}{*}{$(n)^{\mathrm{a}}$} & Length & Holes $^{b}$ \\
\hline & & $\begin{array}{c}\text { Mean } \pm \text { sd } \\
\text { (Range) }\end{array}$ & & $\begin{array}{l}\text { Mean } \pm \text { sd } \\
\text { (Range) }\end{array}$ & $\begin{array}{l}\text { Mean } \pm \text { sd } \\
\text { (Range) }\end{array}$ & & $\begin{array}{c}\text { Mean } \pm \text { sd } \\
\text { (Range) }\end{array}$ & & $\begin{array}{c}\text { Mean } \pm \text { sd } \\
\text { (Range) }\end{array}$ & $\begin{array}{c}\text { Mean } \pm \text { sd } \\
\text { (Range) }\end{array}$ \\
\hline \multicolumn{11}{|l|}{ WMNH-2015-INV-2 } \\
\hline Cup \& knobbed plate & $(137)$ & $\begin{array}{l}108 \pm 38 \\
(33-249)\end{array}$ & $(0)$ & & & (168) & $\begin{array}{l}110 \pm 23 \\
(50-169)\end{array}$ & $(0)$ & & \\
\hline Poorly knobbed plate & $(52)$ & $\begin{array}{l}156 \pm 57 \\
(45-276)\end{array}$ & (97) & $\begin{array}{l}129 \pm 65 \\
(36-341)\end{array}$ & & (16) & $\begin{array}{l}107 \pm 25 \\
(73-156)\end{array}$ & $(68)$ & $\begin{array}{l}109 \pm 34 \\
(47-193)\end{array}$ & \\
\hline Multi-layered knobbed plate & (11) & $\begin{array}{l}285 \pm 63 \\
(185-382)\end{array}$ & $(0)$ & & & $(16)$ & $\begin{array}{l}250 \pm 62 \\
(142-371)\end{array}$ & $(0)$ & & \\
\hline Endplate & $(0)$ & & (4) & $\begin{array}{l}304 \pm 38 \\
(250-335)\end{array}$ & $\begin{array}{l}156 \pm 23 \\
(140-190)\end{array}$ & $(0)$ & & (4) & $\begin{array}{l}96 \pm 20 \\
(71-120)\end{array}$ & $\begin{array}{l}23 \pm 12 \\
(16-40)\end{array}$ \\
\hline \multicolumn{11}{|l|}{ WMNH-2015-INV-4 } \\
\hline Cup \& knobbed plate & (139) & $\begin{array}{l}98 \pm 32 \\
(35-221)\end{array}$ & $(0)$ & & & $(171)$ & $\begin{array}{l}91 \pm 20 \\
(39-134)\end{array}$ & $(0)$ & & \\
\hline Poorly knobbed plate & (51) & $\begin{array}{l}147 \pm 64 \\
(43-288)\end{array}$ & $(88)$ & $\begin{array}{l}131 \pm 59 \\
(42-279)\end{array}$ & & $(15)$ & $\begin{array}{l}72 \pm 21 \\
(38-113)\end{array}$ & $(66)$ & $\begin{array}{l}106 \pm 40 \\
(40-203)\end{array}$ & \\
\hline Multi-layered knobbed plate & (10) & $\begin{array}{l}309 \pm 70 \\
(188-400)\end{array}$ & $(0)$ & & & $(14)$ & $\begin{array}{l}337 \pm 98 \\
(185-511)\end{array}$ & $(0)$ & & \\
\hline Endplate & $(0)$ & & $(5)$ & $\begin{array}{l}326 \pm 27 \\
(294-353)\end{array}$ & $\begin{array}{l}204 \pm 65 \\
(118-280)\end{array}$ & $(0)$ & & (4) & $\begin{array}{l}133 \pm 18 \\
(120-159)\end{array}$ & $\begin{array}{l}39 \pm 8 \\
(32-51)\end{array}$ \\
\hline \multicolumn{11}{|l|}{ WMNH-2015-INV-5 } \\
\hline Cup \& knobbed plate & $(141)$ & $\begin{array}{l}98 \pm 31 \\
(32-195)\end{array}$ & $(0)$ & & & $(172)$ & $\begin{array}{l}93 \pm 20 \\
(39-180)\end{array}$ & $(0)$ & & \\
\hline Poorly knobbed plate & (49) & $\begin{array}{l}150 \pm 58 \\
(39-291)\end{array}$ & $(86)$ & $\begin{array}{l}122 \pm 61 \\
(36-271)\end{array}$ & & $(10)$ & $\begin{array}{l}70 \pm 11 \\
(53-94)\end{array}$ & $(63)$ & $\begin{array}{l}90 \pm 21 \\
(51-155)\end{array}$ & \\
\hline Multi-layered knobbed plate & (10) & $\begin{array}{l}340 \pm 95 \\
(169-445)\end{array}$ & $(0)$ & & & (18) & $\begin{array}{l}309 \pm 105 \\
(156-554)\end{array}$ & $(0)$ & & \\
\hline Endplate & $(0)$ & & $(5)$ & $\begin{array}{l}275 \pm 91 \\
(119-356)\end{array}$ & $\begin{array}{l}199 \pm 73 \\
(98-272)\end{array}$ & $(0)$ & & (4) & $\begin{array}{l}129 \pm 10 \\
(119-142)\end{array}$ & $\begin{array}{l}35 \pm 12 \\
(19-48)\end{array}$ \\
\hline \multicolumn{11}{|l|}{ MNW-11494 (syntype) } \\
\hline Cup \& knobbed plate & \multicolumn{2}{|c|}{ Not measured } & & \multicolumn{2}{|c|}{ Not measured } & $(32)$ & $\begin{array}{c}84 \pm 17 \\
(41-130)\end{array}$ & & \multicolumn{2}{|c|}{ Not measured } \\
\hline Poorly knobbed plate & \multicolumn{3}{|c|}{ Not measured } & \multicolumn{2}{|c|}{ Not measured } & (4) & $\begin{array}{l}98 \pm 14 \\
(89-114)\end{array}$ & & \multicolumn{2}{|c|}{ Not measured } \\
\hline Multi-layered knobbed plate & \multirow{2}{*}{\multicolumn{2}{|c|}{$\begin{array}{l}\text { Not measured } \\
\text { Not measured }\end{array}$}} & & \multirow{2}{*}{\multicolumn{2}{|c|}{$\begin{array}{l}\text { Not measured } \\
\text { Not measured }\end{array}$}} & \multirow{2}{*}{\multicolumn{2}{|c|}{$\begin{array}{l}\text { Only b } \\
(0)\end{array}$}} & & \multirow{2}{*}{\multicolumn{2}{|c|}{$\begin{array}{l}\text { Not measured } \\
\text { Not measured }\end{array}$}} \\
\hline Endplate & & & & & & & & & & \\
\hline
\end{tabular}

${ }^{\text {a }}$ Because ossicles were very abundant and overcrowded, we could not measure them on the basis of random selection; however, value $n$ shows the approximate order of abundance of each ossicle type. As an exception, we selectively measured rare ossicles: endplates, multilayered knobbed plates, and poorly knobbed plates in the dorsal side. ${ }^{\mathrm{b}}$ Counts for endplate holes. Estimation on the basis of a half piece in case the endplate was broken.

39-180 $\mu \mathrm{m}$ (mean 91-110 $\mu \mathrm{m}$ ) in dorsal side (Fig. 3, Table 2); both measurements did not differ significantly among three counted specimens (Kruskal-Wallis test, $P \mathrm{~s}>0.05$ ). Among latter two types, poorly knobbed plates relatively rich in inner layer of integument in abdominal side where plates frequently elongated, and rare in dorsal side where plates not elongated, length 39-291 $\mu \mathrm{m}$ (mean 147-156 $\mu \mathrm{m}$ ) in abdominal side and 38-156 $\mu \mathrm{m}$ (mean 70-107 $\mu \mathrm{m}$ ) in dorsal side (Fig. 3, Table 2). Measurements did not differ significantly among three counted specimens in abdomi- 

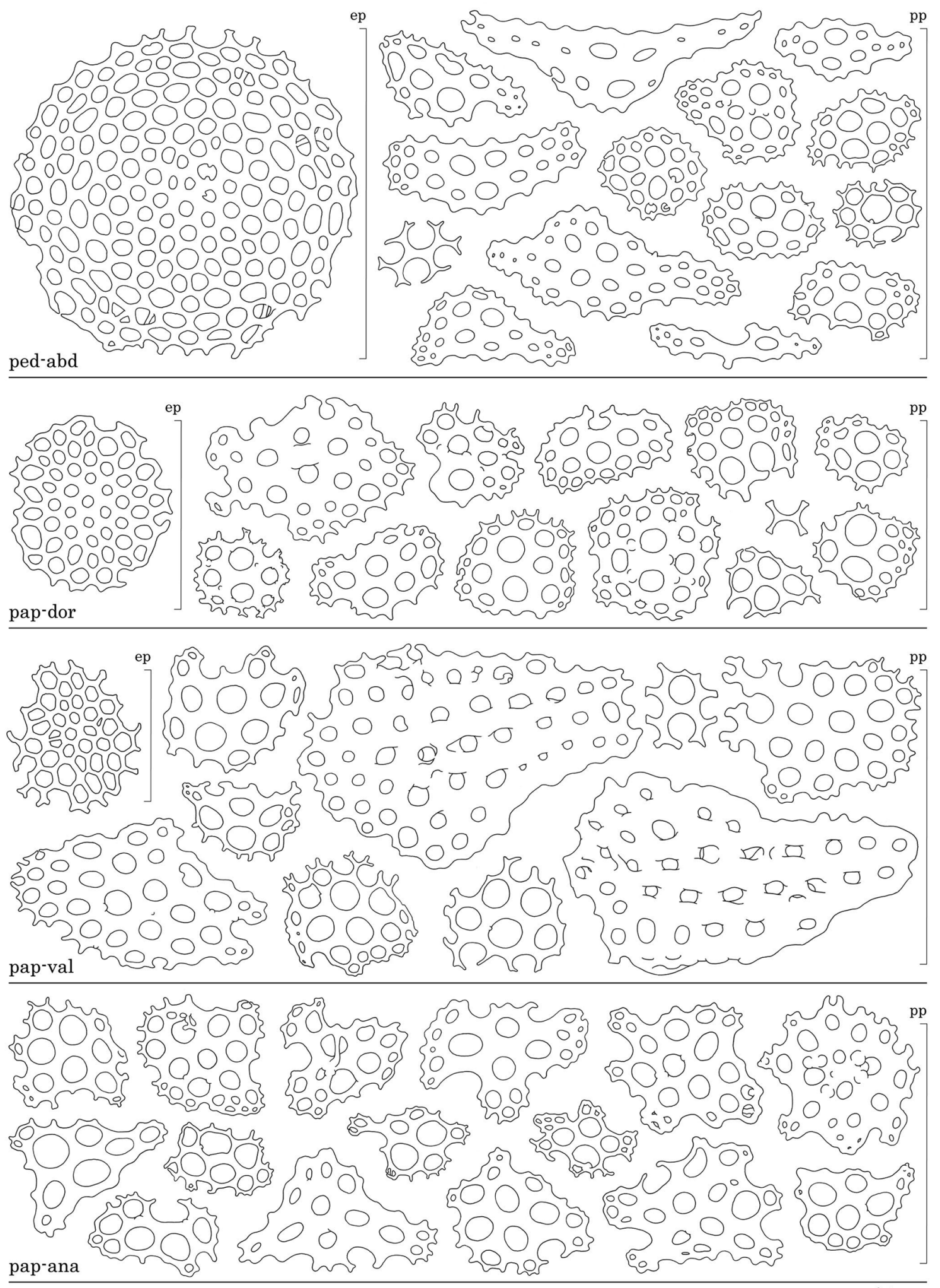

Fig. 4. Plesiocolochirus inornatus (Marenzeller, 1881), ossicles of pedicels in middle part of abdominal side (ped-abd), ambulacral-papillae in dorsal side (pap-dor), ambulacral-papillae in tip of valve (pap-val) of abdominal-lateral side, and ambulacral-papillae in anal (pap-ana) of abdominal-lateral side. Abbreviations: ep, end plate; pp, poorly knobbed plate. WMNH-2015-INV-4. 
nal side (Kruskal-Wallis test, $P>0.05$ ); these ossicles occurred with very low frequency in dorsal side and statistical analysis not applied; multi-layered knobbed plates present in inner layer of integument in both abdominal and dorsal sides, mean length of $285-340 \mu \mathrm{m}$ in abdominal side and 250-337 $\mu \mathrm{m}$ in dorsal side (Fig. 3, Table 2); both measurements did not differ significantly among three counted specimens (Kruskal-Wallis test, $P_{\mathrm{s}}>0.05$ ).

Two types of ossicles found in present samples in both pedicels in abdominal side and ambulacral-papillae in dorsal side: 1) poorly knobbed plates and 2) endplates (Fig. 4, Table 2). Poorly knobbed plates, having weak curvature, and plates frequently elongated in abdominal side and mean length of $122-131 \mu \mathrm{m}$, not elongated in dorsal side and mean length of 90-109 $\mu \mathrm{m}$ (Fig. 4, Table 2). Endplate very weak and fragile, sometimes not complete circular, diameter 119-356 $\mu \mathrm{m}$ (mean 275-326 $\mu \mathrm{m}$ ) with approximately 98-280 holes (mean 156-204) in abdominal side; these ossicles apparently larger than that of ambulacral- papillae where endplates mostly incomplete, diameter 71-159 $\mu \mathrm{m}$ (mean 96-133 $\mu \mathrm{m}$ ) with approximately 16-51 holes (mean 23-39) in dorsal side (Fig. 4, Table 2). Both measurements did not differ significantly among three counted specimens in abdominal side (Kruskal-Wallis test, $P \mathrm{~s}>0.05)$; however, they differed significantly in dorsal side (Kruskal-Wallis test, $P_{\mathrm{S}}<0.05$ ).

Four types of ossicles found in body-wall integument in valve: 1) 'cups' built with an $\mathrm{X}$-shaped central frame and 2) knobbed plates, both mostly from outer layer of integument; these ossicles, irrespective of forms, widely varied in size, length 38-179 $\mu \mathrm{m}$ (mean 70-83 $\mu \mathrm{m}$ ) (Fig. 3, Table 3 ), and measurements differed significantly among three counted specimens (Kruskal-Wallis test, $P<0.01$ ). Other two types mostly from inner layer of integument: 3 ) poorly knobbed plates, length 41-351 $\mu$ m (mean 135-146 $\mu$ m) and 4) multi-layered knobbed plates, mean length of 398$414 \mu \mathrm{m}$ (Fig. 3, Table 3); both measurements did not differ significantly among three counted specimens (Kruskal-

Table 3. Measurements $(\mu \mathrm{m})$ of ossicles from body-wall integument and ambulacral-papillae of oral valve of Plesiocolochirus inornatus (Marenzeller, 1881) (Figs. 3 and 4).

\begin{tabular}{|c|c|c|c|c|c|}
\hline \multirow{4}{*}{ Ossicle type } & \multicolumn{5}{|c|}{ Oral valve (in the abdominal-lateral side) } \\
\hline & \multicolumn{2}{|c|}{ Body-wall integument } & \multicolumn{3}{|c|}{ Ambulacral-papillae } \\
\hline & \multirow{2}{*}{$(n)^{\mathrm{a}}$} & Length & \multirow{2}{*}{$(n)$} & Length & Holes $^{\mathrm{b}}$ \\
\hline & & Mean \pm sd (Range) & & $\overline{\text { Mean } \pm \text { sd (Range) }}$ & \\
\hline \multicolumn{6}{|l|}{ WMNH-2015-INV-2 } \\
\hline Cup \& knobbed plate & $(50)$ & $\begin{array}{c}83 \pm 32 \\
(42-179)\end{array}$ & $(0)$ & & \\
\hline Poorly knobbed plate & $(31)$ & $\begin{array}{l}135 \pm 39 \\
(63-183)\end{array}$ & $(61)$ & $\begin{array}{l}115 \pm 42 \\
(43-213)\end{array}$ & \\
\hline Multi-layered knobbed plate & (19) & $\begin{array}{l}414 \pm 120 \\
(261-616)\end{array}$ & $(0)$ & & \\
\hline Endplate & $(0)$ & & $(0)$ & & \\
\hline \multicolumn{6}{|l|}{ WMNH-2015-INV-4 } \\
\hline Cup \& knobbed plate & $(52)$ & $\begin{array}{c}70 \pm 23 \\
(39-134)\end{array}$ & $(0)$ & & \\
\hline Poorly knobbed plate & $(30)$ & $\begin{array}{l}146 \pm 76 \\
(41-351)\end{array}$ & $(58)$ & $\begin{array}{l}129 \pm 51 \\
(41-313)\end{array}$ & \\
\hline Multi-layered knobbed plate & $(17)$ & $\begin{array}{l}398 \pm 165 \\
(160-828)\end{array}$ & $(0)$ & & \\
\hline Endplate & $(0)$ & & (1) & 141 & 33 \\
\hline \multicolumn{6}{|l|}{ WMNH-2015-INV-5 } \\
\hline Cup \& knobbed plate & $(36)$ & $\begin{array}{c}76 \pm 27 \\
(38-163)\end{array}$ & $(0)$ & & \\
\hline Poorly knobbed plate & $(31)$ & $\begin{array}{l}144 \pm 60 \\
(43-239)\end{array}$ & $(38)$ & $\begin{array}{l}116 \pm 63 \\
(37-281)\end{array}$ & \\
\hline Multi-layered knobbed plate & $(33)$ & $\begin{array}{l}405 \pm 199 \\
(178-923)\end{array}$ & $(0)$ & & \\
\hline Endplate & $(0)$ & & (1) & 99 & 25 \\
\hline
\end{tabular}

${ }^{a}$ Because ossicles were very abundant and overcrowded, we could not measure them on the basis of random selection in the body-wall integument; however, value $n$ shows approximate order of abundance of each ossicle type. ${ }^{\mathrm{b}}$ Counts for endplate holes. 
Table 4. Measurements $(\mu \mathrm{m})$ of ossicles from body-wall integument and ambulacral-papillae in the anal periphery of Plesiocolochirus inornatus (Marenzeller, 1881) (Figs. 3 and 4).

\begin{tabular}{|c|c|c|c|c|}
\hline \multirow{4}{*}{ Ossicle type } & \multicolumn{4}{|c|}{ Anal periphery (in the abdominal-lateral side) } \\
\hline & \multicolumn{2}{|c|}{ Body-wall integument } & \multicolumn{2}{|c|}{ Ambulacral-papillae } \\
\hline & \multirow{2}{*}{$(n)^{\mathrm{a}}$} & Length & \multirow{2}{*}{$(n)$} & Length \\
\hline & & Mean \pm sd (Range) & & Mean \pm sd (Range) \\
\hline \multicolumn{5}{|l|}{ WMNH-2015-INV-2 } \\
\hline Cup \& knobbed plate & $(27)$ & $\begin{array}{c}87 \pm 29 \\
(45-136)\end{array}$ & $(0)$ & \\
\hline Poorly knobbed plate & $(10)$ & $\begin{array}{l}120 \pm 35 \\
(61-161)\end{array}$ & $(36)$ & $\begin{array}{l}122 \pm 33 \\
(41-200)\end{array}$ \\
\hline Multi-layered knobbed plate & $(10)$ & $\begin{array}{l}345 \pm 96 \\
(215-500)\end{array}$ & $(0)$ & \\
\hline \multicolumn{5}{|l|}{ WMNH-2015-INV-4 } \\
\hline Cup \& knobbed plate & (29) & $\begin{array}{c}89 \pm 21 \\
(42-130)\end{array}$ & $(0)$ & \\
\hline Poorly knobbed plate & $(15)$ & $\begin{array}{l}107 \pm 40 \\
(50-203)\end{array}$ & $(36)$ & $\begin{array}{l}132 \pm 27 \\
(88-178)\end{array}$ \\
\hline Multi-layered knobbed plate & (13) & $\begin{array}{l}352 \pm 158 \\
(173-641)\end{array}$ & $(0)$ & \\
\hline \multicolumn{5}{|l|}{ WMNH-2015-INV-5 } \\
\hline Cup \& knobbed plate & $(48)$ & $\begin{array}{c}85 \pm 28 \\
(36-145)\end{array}$ & $(0)$ & \\
\hline Poorly knobbed plate & (29) & $\begin{array}{l}131 \pm 53 \\
(50-272)\end{array}$ & (13) & $\begin{array}{l}119 \pm 16 \\
(91-150)\end{array}$ \\
\hline Multi-layered knobbed plate & $(26)$ & $\begin{array}{l}328 \pm 161 \\
(141-788)\end{array}$ & $(0)$ & \\
\hline
\end{tabular}

\footnotetext{
${ }^{a}$ Because ossicles were very abundant and overcrowded, we could not measure them on the basis of random selection in the body-wall integument; however, value $n$ shows approximate order of abundance of each ossicle type.
}

Wallis test, $P \mathrm{~s}>0.05)$.

Two types of ossicles found in ambulacral-papillae at valve tip: 1) poorly knobbed plates, larger plates thickening and having weak curvature, widely varied in size, length 37-313 $\mu \mathrm{m}$ (mean 115-129 $\mu \mathrm{m}$ ); measurements did not differ significantly among three counted specimens (Kruskal-Wallis test, $P>0.05$ ); 2) endplates, incomplete and frequently lacking, only two endplates observed, diameter $99 \mu \mathrm{m}$ with 25 holes and diameter $141 \mu \mathrm{m}$ with 33 holes (Fig. 4, Table 3); statistical analysis not applied.

Four types of ossicles found in body-wall integument around anal: mostly 1) 'cups' built with an $\mathrm{x}$-shaped central frame and 2) knobbed plates; these ossicles, irrespective of forms, widely varied in size, length $36-145 \mu \mathrm{m}$ (mean $85-89 \mu \mathrm{m}$ ) (Fig. 3, Table 4); measurements did not differ significantly among three counted specimens (KruskalWallis test, $P>0.05$ ). Other two types 3 ) poorly knobbed plates, length 50-272 $\mu \mathrm{m}$ (mean 107-131 $\mu \mathrm{m}$ ) and 4) multilayered knobbed plates, including 'scales' covering body surface of anal periphery, mean length of 328-352 $\mu \mathrm{m}$ (Fig. 3, Table 4); both measurements did not differ significantly among three counted specimens (Kruskal-Wallis test, $P \mathrm{~s}>0.05)$.

One type of ossicle found in papillae in anal region: 1) poorly knobbed plates, mean length of 119-132 $\mu \mathrm{m}$ (Fig. 4, Table 4); measurements did not differ significantly among three counted specimens (Kruskal-Wallis test, $P>0.05$ ). No endplate confirmed in any of three counted specimens.

Three types of ossicles found in tentacle: 1) perforated rods, some arc-shaped and some flattened, widely varied in size, length $15-841 \mu \mathrm{m}$ (mean 301-397 $\mu \mathrm{m}$ ) (Fig. 5, Table 5); measurements did not differ significantly among three counted specimens (Kruskal-Wallis test, $P>0.05$ ). Larger rods very thickened and having many knobs or blunt spines. 2) Small 'rosette'-like indeterminate-shaped ossicles, length 22-79 $\mu \mathrm{m}$ (mean 44-58 $\mu \mathrm{m}$ ), and 3) plates, length 20-331 $\mu \mathrm{m}$ (mean 111-152 $\mu \mathrm{m}$ ) (Fig. 5, Table 5). Both measurements did not differ significantly among three counted specimens (Kruskal-Wallis test, $P \mathrm{~s}>0.05$ ).

Three types of ossicles found in peri-oral skin and middle part of introvert: 1) small 'rosette-like indeterminateshaped ossicles and 2) small 'cups' built with an x-shaped central frame; these ossicles, irrespective of forms, mean length of $40-56 \mu \mathrm{m}$ in peri-oral skin, $52-53 \mu \mathrm{m}$ in mid- 

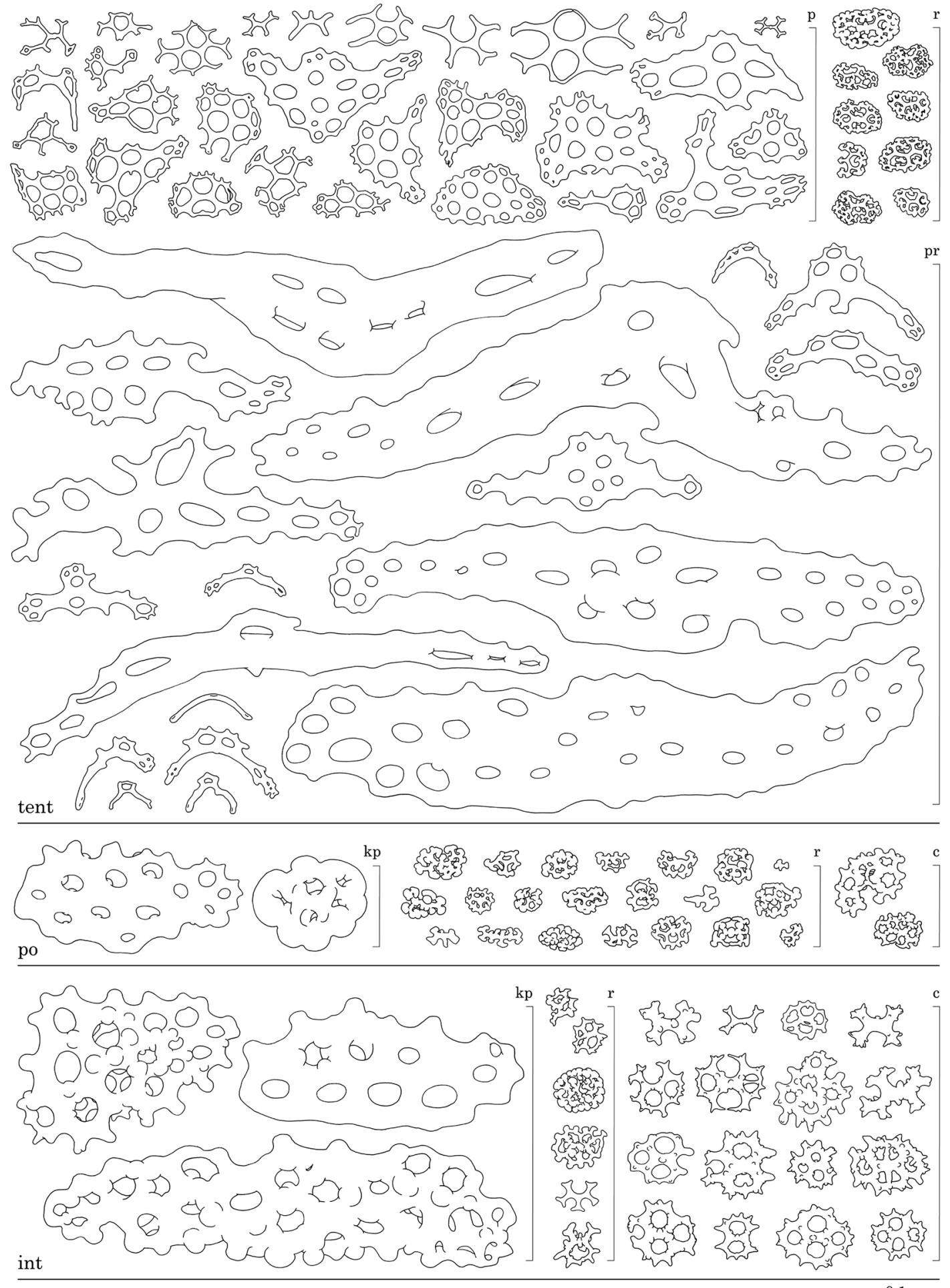

Fig. 5. Plesiocolochirus inornatus (Marenzeller, 1881), ossicles of tentacle (ten), peri-oral skin (po), and middle part of introvert (int) on the abdominal-lateral side. Abbreviations: c, cup; kp, knobbed plate; p, plate; pr, perforated rod; r, rosette. WMNH-2015-INV-4.

dle part of introvert (Fig. 5, Table 5); measurements in peri-oral skin differed significantly among three counted specimens (Kruskal-Wallis test, $P<0.01$ ) and did not differ in middle part of introvert (Kruskal-Wallis test, $P>0.05$ ). In addition, 3) knobbed plates, very thickened and some- times having many knobs or blunt spines, mean length of 94-160 $\mu \mathrm{m}$ in peri-oral skin, $123-192 \mu \mathrm{m}$ in middle part of introvert (Fig. 5, Table 5), occurring with very low frequency; statistical analysis not applied. 
Table 5. Measurements $(\mu \mathrm{m})$ of ossicles from tentacle, peri-oral skin, and middle part of introvert of Plesiocolochirus inornatus (Marenzeller, 1881) in the abdominal-lateral side (Fig. 5).

\begin{tabular}{|c|c|c|c|c|c|c|}
\hline \multirow{3}{*}{ Ossicle type } & \multicolumn{2}{|c|}{ Tentacle } & \multicolumn{2}{|c|}{ Peri-oral skin } & \multicolumn{2}{|c|}{ Introvert } \\
\hline & \multirow{2}{*}{$(n)^{\mathrm{a}}$} & Length & \multirow{2}{*}{$(n)$} & Length & \multirow{2}{*}{$(n)$} & Length \\
\hline & & Mean \pm sd (Range) & & Mean \pm sd (Range) & & Mean \pm sd (Range) \\
\hline \multicolumn{7}{|l|}{ WMNH-2015-INV-2 } \\
\hline Perforated rod & $(81)$ & $\begin{array}{l}397 \pm 155 \\
(51-782)\end{array}$ & $(0)$ & & $(0)$ & \\
\hline Rosette (\& cup) & (6) & $\begin{array}{l}50 \pm 7 \\
(41-62)\end{array}$ & $(65)$ & $\begin{array}{l}49 \pm 13 \\
(22-94)\end{array}$ & $(54)$ & $\begin{array}{l}53 \pm 12 \\
(20-81)\end{array}$ \\
\hline Plate & $(22)$ & $\begin{array}{l}152 \pm 97 \\
(20-278)\end{array}$ & $(0)$ & & $(0)$ & \\
\hline Knobbed plate & $(0)$ & & (1) & $\begin{array}{c}94 \\
(94)\end{array}$ & (13) & $\begin{array}{l}123 \pm 14 \\
(102-152)\end{array}$ \\
\hline \multicolumn{7}{|l|}{ WMNH-2015-INV-4 } \\
\hline Perforated rod & $(68)$ & $\begin{array}{r}349 \pm 198 \\
(84-748)\end{array}$ & $(0)$ & & $(0)$ & \\
\hline Rosette (\& cup) & (9) & $\begin{array}{l}58 \pm 6 \\
(49-68)\end{array}$ & (29) & $\begin{array}{l}40 \pm 17 \\
(21-83)\end{array}$ & $(51)$ & $\begin{array}{l}53 \pm 11 \\
(36-82)\end{array}$ \\
\hline Plate & $(36)$ & $\begin{array}{l}123 \pm 73 \\
(20-267)\end{array}$ & $(0)$ & & $(0)$ & \\
\hline Knobbed plate & $(0)$ & & (3) & $\begin{array}{l}160 \pm 52 \\
(101-198)\end{array}$ & (3) & $\begin{array}{l}192 \pm 74 \\
(110-254)\end{array}$ \\
\hline \multicolumn{7}{|l|}{ WMNH-2015-INV-5 } \\
\hline Perforated rod & $(64)$ & $\begin{array}{r}301 \pm 228 \\
(15-841)\end{array}$ & $(0)$ & & $(0)$ & \\
\hline Rosette (\& cup) & (9) & $\begin{array}{l}44 \pm 20 \\
(22-79)\end{array}$ & $(40)$ & $\begin{array}{l}56 \pm 14 \\
(33-83)\end{array}$ & (53) & $\begin{array}{l}52 \pm 16 \\
(25-88)\end{array}$ \\
\hline Plate & $(36)$ & $\begin{array}{l}111 \pm 67 \\
(22-331)\end{array}$ & $(0)$ & & $(0)$ & \\
\hline Knobbed plate & $(0)$ & & (2) & $\begin{array}{c}142 \\
(140-143)\end{array}$ & (7) & $\begin{array}{l}153 \pm 83 \\
(91-338)\end{array}$ \\
\hline
\end{tabular}

\footnotetext{
${ }^{a}$ Because ossicles were very abundant and overcrowded, we could not measure them on the basis of random selection in the tentacle; however, value $n$ shows the approximate order of abundance of each ossicle type.
}

\section{Comparative material}

MNW-11494, syntype (length $65 \mathrm{~mm}$, width $19 \mathrm{~mm}$ ), Naturhistorisches Museum Wien, collected by Dr. Roretz, 1876, Japan. Although body colour is different, external and internal morphologies of body, appendages, tentacles, calcareous ring, Polian vesicle, stone canal, and gonad, conform well to six additional specimens found recently. In the body-wall integument in mid-dorsal side, four types of ossicles were found (Fig. 6, Table 2): 1) 'cups' built with an x-shaped central frame, 2) knobbed plates and knobbed button, 3) poorly knobbed plates, 4) and broken pieces of multi-layered knobbed plates (not measured). Because only a minute tissue sample was examined, statistical analysis of the ossicle composition was not performed, however, shape and length of these ossicles conformed well to present new specimens.

\section{Distribution}

Japan (Marenzeller 1881), 10.8-25.2 m depth in Tokyo Bay and $39.6 \mathrm{~m}$ depth in Negishi Bay near Yokohama, mid-eastern Japan (Mitsukuri 1912), Kyushu, southern Japan (Ohshima 1912), China (Liao and Clark 1995), Myanmar (Pearson 1910), and 5-6 m depth in the Pacific Ocean, the south coast of Fukushima Prefecture, eastern Japan. This animal is a very common and dominant species in the sampling site, and there was an infinite number of individuals openly attached to the quay wall over a wide area, sometimes showing crowding.

\section{Remarks}

The morphological account provided by Marenzeller (1881) was so precise and accurate that our observation yielded no taxonomic novelty. However, the general appearance of the body and calcareous ring and ossicle mor- 


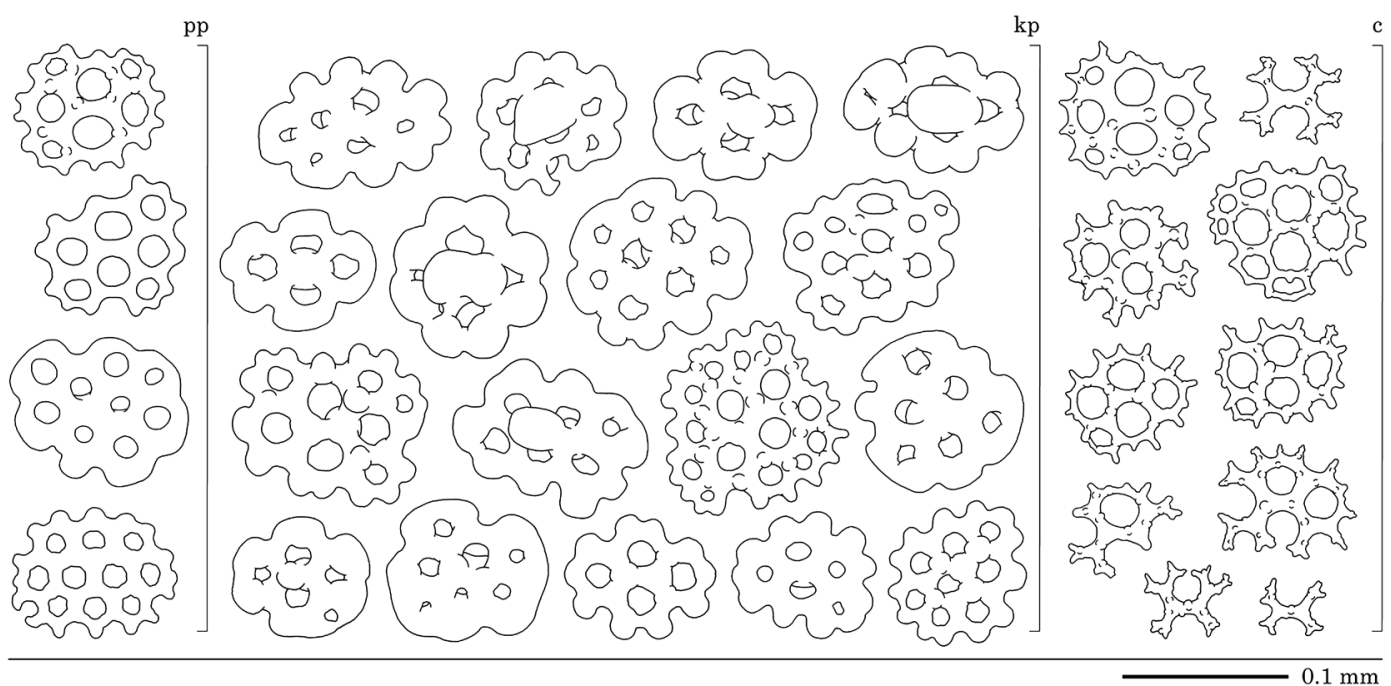

Fig. 6. Syntype (MNW-11494) of Plesiocolochirus inornatus (Marenzeller, 1881), ossicles of body-wall integument in middle part of dorsal side. Abbreviations: c, cup; kp, knobbed plate; pp, poorly knobbed plate.

phology (Figs. 1-5), for which sufficient information is unavailable in the literature, may be useful for the identification of this species.

\section{Discussion}

The family Cucumariidae Ludwig, 1894, is the largest family of dendrochirotid holothurians, and it currently includes 66 genera (Paulay, 2014). In this family, several subfamilies have been regarded as significant, such as $\mathrm{Cu}$ cumariinae Ludwig, 1898; Colochirinae Panning, 1949; Thyonidiinae Heding and Paning, 1954; Ypsilothuriinae, Heding, 1942. However, the differences between $\mathrm{Cu}-$ cumariinae and Colochirinae are unclear because there is only about one feature difference in the original diagnosis; "Körbchen" (basket-like ossicles) are present in the body integument of Colochirinae but not Cucumariinae (Panning 1949); since then, it has become clear that there are various shapes of basket-like ossicles. For the existence of basket-like ossicles in a genus, the explanation differs from researcher to researcher. Therefore, it is difficult to draw a definite line of demarcation between Cucumariinae and Colochirinae in the literature, and both subfamilies are currently composed of 46 genera. Thus, there are neither practical monographs nor taxonomic keys to determine what species should be included, and we had to carefully examine the original descriptions for many related species.

Fortunately, Marenzeller (1881) provided a marvellously detailed description for P. inornatus (Marenzeller 1881, as Colochirus inornatus) on the basis of many minute internal and external body observations and ossicle observations. The diagnosis is summarized as follows (several sentences were in the diagnosis of the genus): largest individual of $50 \mathrm{~mm}$ length, contracting, cylindrical body, body cross-section angulated lightly in each radius; body broadest at middle of $20 \mathrm{~mm}$ breadth; body bent upwards, posterior end sharply standing out; dorsal colour uneven light grey, and slightly lighter colour at points where ambulacural-papillae are scattered; ventral plane wide, however, not reaching both body ends, colourless, with three longitudinal series of ventral pedicels; each series broadest at its middle, and composed of about five pedicels in breadth, gradually thinner towards both ends, where pedicels composed of a double row; body anterior closed by five valvular projections, each valve tip with ambulacuralpapillae, which colour blurred with black; tentacles ten, light colour, ventral two much smaller; body scales imbricate in posterior end, usual body scales not present in other regions; five small anal scales present; one stone canal and one Polian vesicle; tubules of gonads unbranched, long as half of body length; calcareous ring having no posterior projection; ossicles in surface layer of body integument display almost flat cups with knobs or spines; cups built with an $\mathrm{x}$-shaped central frame, with branched arms, arms partly connected with each other or partly free; ossicles in inner layer of body integument display thick and robust knobbed buttons with 4-6 or more holes; multilayered plates (usual scales) not excessively large, mostly $0.3-0.7 \mathrm{~mm}$ length, $1 \mathrm{~mm}$ or more at body end; ossicles in pedicels display broad rod-like plates, lightly knobbed, not so long (Marenzeller 1881, originally in German).

Although the body size and colour of the syntype are different to those of the recent specimens (the differences are presumed to be due to change over time), our present observations are mostly consistent with this diagnosis, with an exception for the posterior projection, which slightly observed in this study, however, not long like a normal posterior projection of phyllophorid species. Further, on the basis of observations of our specimens, we obtained new information on the external and ossicle morphology of this species. In the living state, the body has obviously distinguishable dorsal and ventral sides, mid- 
body of the ventral side is extremely flattened and nearly half-round in the cross-section, and the dorsal surface is smooth with retracted minute ambulacral-papillae scattered uniformly regardless of radii or inter-radii. Ossicles are of quite variable shape, even ossicles obtained from the same tissue sample. Most ossicles are shallow spinous cups and their derivatives (knobbed plates) in the bodywall integument, poorly knobbed plates in the pedicels and papillae, and perforated rods in the tentacles. Shallow spinous cups are built with an $\mathrm{x}$-shaped central frame, with branched arms connected marginally with each other or partly free. The arms are sometimes bridged internally; however, the cups with bridging between the margin and center (i.e. 'basket') are very rare and incomplete.

Measurements of ossicles differed significantly among the three counted specimens at the following sites: all four types of ossicles in ambulacral-papillae in the middle part of the dorsal side and two types of ossicles in the bodywall integument in the valve. Measurement of ossicles from these sites was apparently not suitable for identifying this species, and we recommend measuring ossicles from the body-wall integument and pedicels in the middle part of the abdominal side, where measurements of ossicles did not show any significant differences (at least in our three specimens).

Currently, this species has been included in the genus Plesiocolochirus Cherbonnier, 1946 (type species: Holothuria spinosa Quoy and Gaimard, 1834) by Clark and Rowe (1971).

Diagnosis of the genus is summarized as follows: body barrel-shaped, bend upward; tentacles 10, well branched, ventral two small; five valvular projections in body anterior, each valve with 5-10 ambulacral-papillae; pedicels mostly contain well-developed endplates in ventral side, only rudimentary endplates or lacking in dorsal side; anal end protrudes like a chimney, with imbrications of body scales; 5-10 small anal scales present; inter-radial element of calcareous ring with fine tapering anterior projection, and deep concaving posterior notch; radial element of calcareous ring with forked anterior projection, and two posterior prolongations; ossicles mostly large irregular knobbed buttons with 4-12 holes, and large "baskets" built with an X-shaped central frame, or small "baskets" without an $\mathrm{x}$-shaped central frame; ossicles in pedicels robust "rods" with perforations in extremities; ossicles in tentacles large "rods", numerous, bowed, or small "rods" with branching two or three times and with perforations in extremities (Cherbonnier 1946, originally in French).

Our specimens, however, did not show complete "baskets" in any of the specimens examined. It became clear that the shapes of basket-like ossicles are full of variety; therefore, the feature of the ossicles "built with an $\mathrm{x}$-shaped central frame" can be considered to have significant meaning. This conforms well for the present species. In addition, the robust "rods" of pedicels with perforations in the extremities can be considered as the same as that of the poorly knobbed plates in the abdominal side where the plates are elongated. In conclusion, it was found that the features of $P$. inornatus were mostly consistent with the diagnosis, and this species could still belong to the genus Plesiocolochirus, although the body form of the present specimens is unique.

\section{Acknowledgements}

We are grateful to Dr Sattmann Helmut and Mr Szeiler Stefan for providing us the opportunity to examine the syntype of $P$. inornatus deposited in MNW, Dr. Hendrik Freitag, and Mr. Yukimitsu Imahara for providing many help. We would like to thank the entire staff of the Environmental Aquarium Aquamarine Fukushima and Misaki Marine Biological Station, School of Science, University of Tokyo, for the invaluable assistance provided during this study. We also thank two anonymous reviewers for reviewing the manuscript, and to Dr. François Michonneau for checking the English of this text. This work was partially supported by the Research Institute of Marine Invertebrates (Tokyo) 2015KO-1. The financial support for a portion of the microscopic observation was provided by $\mathrm{Mr}$ Shinichi Ikebe of the Supporters' Association of WMNH.

\section{References}

Chang FY (1937, in press 1948) Echinoderms of Tsingtao. Contribution from the institute of Zoology National Academy of Peiping Vol. IV, No. 2: 33-103.

Cherbonnier G (1946) Sur une Holothurie de Quoy et Gaimard, type d'un nouveau genre: Plesiocolochirus n.g. Bulletin du Muséum National Histoire Naturelle $2^{\mathrm{e}}$ série, Tome XVIII, $\mathrm{n}^{\circ}$ 3: 280-286.

Clark AM, Rowe FWE (1971) Holothurioidea. In: Monograph of shallow-water Indo-West Pacific Echinoderms (eds Clark AM, Rowe FWE). Trustees of The British Museum (Natural History), London, pp. 171-210.

Imaoka T (1995) Holothuroidea. In: Genshoku Kensaku Nihon Kaigan Doubutsu Zukan. Vol. II. [Guide to Seashore Animals of Japan with Color Pictures and Keys. Vol. II.] (eds Nishimura S). Hoikusha, Osaka, pp. 553-572. (In Japanese)

Jangoux M, De Ridder C (1990) Annotated catalogue of recent echinoderm type specimens in the collection of the Naturhistorisches Museum Wien. Annotationes Naturhistorisches Museum Wien 91B: 205-213.

Lampert K (1885) Die Seewalzen (Holothurioidea) eine systematische monographie. In: Holothurien. Reisen im Archipel der Philippinen, Teil 2, Wissenschaftliche Resultate (eds Semper C). C. W. Kreidel, Wiesbaden, Bd. 4,3. pp. 1-312.

Liao Y, Clark AM (1995) Class Holothurioidea. In: The Echinoderms of Southern China (eds Liao Y, Clark AM). Science Press, Beijing, pp. 417-545.

Marenzeller E (1881, in press 1882) Neue Holothurien von Japan and China. Verhandungen der Zoologisch-Botanischen Gesellschaft in Wien 31: 121-140.

Mistukuri K (1912) Studies on Actinopodous Holothurioidea. 
The Journal of the College of Science, Imperial University of Tokyo 29: 1-284.

Ohshima H (1914, in press 1915) Report on the holothurians collected by the United States Fisheries Steamer "Albatross" in the Northwestern Pacific during the summer of 1906. Proceedings of the United States National Museum 48: 213-291.

Ohshima H (1918) Holothuroidea in northwest Pacific. XX. Dobutsugaku zasshi 31: 139-149. (in Japanese)

Panning A (1949) Versuch einer Neuordnung der Familie Cucumariidae (Holothurioidea, Dendrochirota). Zoologsche Jahrbücher, Abteilung für Systematik, Ökologie und Geographie Thiere 78: 404-470.

Paulay G (2014) Cucumariidae Ludwig, 1894. Available at: World
Register of Marine Species at http://www.marinespecies.org/ aphia.php? $\mathrm{p}=$ taxdetails\&id=123187 (28 January 2015).

Pearson J (1910) Marine Fauna: Mergui Archipelago, Lower Burma. Collected by Jas. J. Simpson, M. A., B. Sc., and R. N. Rudmose-Brown, B. Sc., University of Aberdeen, February 1907-May 1907: Holothurioidea. Proceedings of The Zoological Society 1910 (January-March): 183-194.

Théel Hj (1886) Report on the Holothurioidea dredged by H. M. S. Challenger during the years 1873-76. Report of the scientific results of the Exploring voyage of H.M.S. Challenger during the years 1873-76. Zoology 39: 1-290.

Yang PF (1937) Report on the holothurians from the Fukien Coast. Bulletin Marine Biology of Amoy China 2: 1-46. 